\title{
Mechanical imperfections effect on the minimum volume stability limit of liquid bridges
}

\author{
M. Gómez, I. E. Parra, and J. M. Perales \\ E.T.S.I. Aeronánticos, Lninersidad Politécnica de Madrid, Pza. Cardenal Cisneros, 3, 28040 Madrid, Spain
}

\begin{abstract}
The bifurcation to unstable equilibrium shapes in the neighborhood of the minimum volume stability limit of liquid bridges has been described by using the Lyapunov-Schmidt technique. Prior to the bifurcation analysis. the stability limits of axisymmetric liquid bridges (both that of maximum and that of minimum volume) have been analytically calculated when the liquid bridge supports are two circular, coaxial disks. The interface shapes have been parametrically described and the parameters corresponding to the marginally stable shapes have been determined in terms of elliptic variables. Bifurcation equations have been obtained analytically describing the behavior near the critical points previously calculated and the effect of small axisymmetric imperfections has been considered. The considered imperfections are inequality in the diameter of the supporting disks, small body forces due to an axial gravity, and liquid bridge rotation as a solid body.
\end{abstract}

\section{INTRODUCTION}

Most of the investigations concerning liquid bridge statics are connected with the study of the stability of axisymmetric equilibrium shapes of liquid bridges with respect to axisymmetric perturbations. ${ }^{1-6}$ More recent results have been reported on the possible loss of stability due to nonaxisymmetric perturbations, ${ }^{1.7-10}$ which are the critical ones in many cases. In a recent paper Slobozhanin et al. ${ }^{11}$ have analyzed the character of the bifurcation of liquid bridges when no imperfections are considered. From all these studies, the interest in determining the stability limits of axisymmetric liquid bridges' equilibrium shapes is obvious. Several methods have been used in order to compute the minimum volume stability limit of long liquid columns under ideal conditions (when neither gravity nor solid body rotation is considered and the disk radii are equal).

To continue with the bifurcation analysis, a detailed description of the constant volume stability limits in the general case, considering not only the maximum volume stability limit but also the minimum volume stability limit corresponding to short liquid columns (for long and medium ones, the limits have been already calculated in previous works, Gillete and Dyson, ${ }^{2}$ Meseguer, ${ }^{12}$ Gómez et al ${ }^{13}$ ). is included. The possibility of stability loss with respect to perturbations of arbitrary shape (not necessarily axisymmetric) is analyzed.

Moreover, concerning the minimum volume stability limit, recent investigations have analyzed the influence of Bond number (Slobozhanin and Perales ${ }^{3}$ ), the Weber number (Slobozhanin and Perales ${ }^{4}$ ). electric fields (González et $a l^{14}$ ). external axial flows (Lowry and Steen ${ }^{15}$ ), and disk diameter inequality (Slobozhanin et al..$^{5}$ ) considering these effects alone and more recently the combined effect of sev- eral of them. ${ }^{16-19}$ However in all these works the problem is finally solved by numerical methods and some calculation difficulties appear when small values of the perturbations are considered. Other studies (Martínez and Perales ${ }^{6}$ ) use a formulation in terms of elliptic functions in order to calculate the minimum volume stability limit in the absence of external forces and in other works, numerical methods are used to compute the stability limits (Perales et al. ${ }^{20}$ Martinez et $a l^{21}$ ). It is very difficult to determine from the abovementioned studies the behavior for small values of the imperfections $(B, H$, and $W)$ and the character of the bifurcation with these imperfections has not been analyzed. Some analytical studies of the bifurcation have been done in the neighborhood of the cylinder (see. e.g.. Vega and Perales ${ }^{22}$ ). but there is little knowledge of this behavior except for this particular configuration. This lack of data and the need for a study of the asymptotic belhavior of the stability limits for small values of the imperfections lead to the use of methods employed in the bifurcation theory in order to analyze the problem in detail.

\section{PROBLEM FORMULATION AND EQUILIBRIUM SHAPES}

The fluid configuration here considered consists of an isothermal mass of liquid of volume $\mathbf{V}$ held by surface tension forces between two parallel coaxial solid disks (of radii $\mathbf{R}_{1}$ and $\mathbf{R}_{2}$, respectively) placed a distance $\mathbf{L}$ apart (see Fig. 1). Such configuration can be uniquely defined by the following dimensionless parameters: the slenderness $\Lambda$ $=\mathbf{L} / 2 \mathbf{R}_{0}$, the dimensionless volume $v=\mathbf{V} / \pi \mathbf{R}_{i v}^{2} \mathbf{L}$. the dimensionless disk radii difference $H=\left(\mathbf{R}_{2}-\mathbf{R}_{1}\right) /\left(\mathbf{R}_{2}+\mathbf{R}_{1}\right)$, the Bond number $B=\Delta \rho \mathbf{g} \mathbf{R}_{0}^{2} / \sigma$, and the Weber number $W$ $=\Delta \rho \mathbf{R}_{0}^{3} \mathbf{\Omega}^{2} / \sigma$. The characteristic magnitudes are: $\mathbf{R}_{0}=\left(\mathbf{R}_{1}\right.$ 


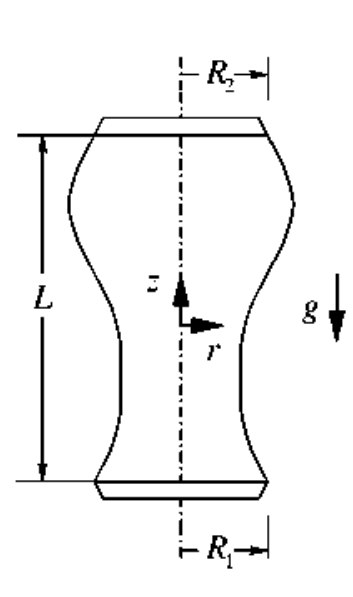

$$
\begin{aligned}
& \Lambda=\frac{L}{2 R_{0}} \\
& V=\frac{V}{\pi R_{0}^{2} L} \\
& H=\frac{R_{0}-R_{1}}{R_{2}-R_{1}} \\
& B=\frac{\Delta \rho g R}{\sigma} \\
& W=\frac{\Delta \rho \Omega^{2} R_{0}^{3}}{\sigma} \\
& R_{0}=\frac{R_{;} R_{1}}{2}
\end{aligned}
$$

FIG. 1. Liguid bridge geometry.

$\left.+\mathbf{R}_{2}\right) / 2 ; \Delta \boldsymbol{\rho}$, the density difference between the liquid bridge and the surrounding media; $g$, the axial acceleration: $\boldsymbol{\Omega}$ the solid body rotation rate; and $\boldsymbol{\sigma}$ the surface tension.

The equilibrium shape of a liquid bridge is described in cylindrical coordinates by the expression $F=F(z, \theta)$. where $F$ is the distance of the liquid interface to the common disks axis, $z$ is the position of the interface point measured along the axis, and $\theta$ is the azimuth angle. Such a surface is described by the Young-Laplace equilibrium equation, which in dimensionless variables reads

$$
M(F)-B z+\frac{W}{2} F^{2}+P=0 .
$$

The operator $M(F)$ is twice the interface mean curvature. Moreover, the system formed by the liquid and its solid circular supports has been considered rotating around its axis as a solid body with an uniform angular velocity $\mathbf{\Omega}$

Equation (I) must be completed with anchoring conditions of the interface to the disk edges, periodicity conditions, and a condition of liquid volume conservation:

$$
\begin{aligned}
& F( \pm \Lambda, \theta)=1 \pm H, \quad \theta \in[0,2 \pi], \\
& F(z, \theta)=F(z, \theta+2 \pi), \quad z \in[-\Lambda, \Lambda], \quad \theta \in[0.2 \pi[, \\
& v=\frac{1}{4 \pi \Lambda} \int_{-\Lambda}^{\Lambda} \int_{0}^{2 \pi} F^{2}(z, \theta) d z d \theta .
\end{aligned}
$$

To write down the above-given expressions all lengths have been made dimensionless with $R_{0} . P$ is a still unknown constant related with the pressure level, which has been made dimensionless with $\sigma / R_{0}$. The parameters $\Lambda, B, H, W$, and $v$ were defined previously.

For a liquid bridge at rest, in the absence of external forces, surrounded by another fluid with constant interface tension. the axisymmetric equilibrium shapes are given by Eq. (I) with $W=B=0$. That is, the equilibrium shape is a surface of revolution of constant mean curvature

$$
M(F)+P=-\frac{1}{F\left(1+F_{z}^{2}\right)^{1 / 2}}+\frac{F_{z z}}{F\left(1+F_{z}^{2}\right)^{3 / 2}}+P=0 .
$$

As is well-known, ${ }^{2,6,23,24}$ the general solution of Eq. (5) can be written in the following parametric form:

$$
\begin{aligned}
& F=\sqrt{\alpha} \sqrt{1-\sin ^{2} a \sin ^{2} \varphi} . \\
& z=\sqrt{\alpha}[\mathrm{F}(a, \varphi) \cos a+\mathrm{E}(a, \varphi)+b] .
\end{aligned}
$$

where $a$ and $b$ are constants $(0 \leqslant a \leqslant \pi), \mathrm{F}(a, \varphi)$ and $\mathrm{E}(a, \varphi)$ are the elliptic integrals of first and second kind and the positive parameter $\alpha$ is defined as

$$
\alpha=\frac{4}{P^{2}(1+\cos a)^{2}} .
$$

The meridian curve generating the revolution surface must then be a piece of an axially periodic Plateau curve: cylinder. unduloids, catenoids, nodoids, and sphere. All of these, except catenoids. can be expressed in the above-given parametric form, ${ }^{2,6,23,24}$ where $a$ identifies the Plateau curve ( $|\cos a|$ is the ratio of hollow-to-summit radii; $0 \leqslant a \leqslant \pi / 2$ and $\pi / 2 \leqslant a \leqslant \pi$ correspond to unduloids and nodoids, respectively) and $\varphi$ is the parameter that makes the curve being run. Actually, parameter $\varphi$ is proportional to Plateau curves' arclength. $d s= \pm \sqrt{\alpha}(1+\cos a) d \varphi$. which is the representation parameter directly used by most authors (see, e.g. Slobozhanin et al. ${ }^{11}$ Lowry and Steen ${ }^{25}$ ).

Using the variable $u=\mathrm{F}(a, \varphi)$, expressions (6) can be written as follows:

$$
F=\sqrt{\alpha} \operatorname{dn} u, \quad z=\sqrt{\alpha}[u \cos a+\mathrm{E}(a, \operatorname{am} u)+b],
$$

where am $u$ and $\operatorname{dn} u$ are the Jacobian elliptic functions anplitude and delta amplitude, respectively. In terms of this solution, the anchoring conditions (2) are then rewritten as

$$
\begin{aligned}
& 1+H=\sqrt{\alpha} \operatorname{dn} u_{1} . \\
& \Lambda=\sqrt{\alpha}\left[u_{1} \cos a+\mathrm{E}\left(a, \operatorname{am} u_{1}\right)+b\right], \\
& 1-H=\sqrt{\alpha} \operatorname{dn} u_{2} . \\
& -\Lambda=\sqrt{\alpha}\left[u_{2} \cos a+\mathrm{E}\left(a, \operatorname{am} u_{2}\right)+b\right] .
\end{aligned}
$$

and the volume conservation requirement as

$$
\begin{aligned}
v= & \frac{\alpha^{3 / 2}}{6 \Lambda}\left\{\sin ^{2} a\left[\operatorname{dn} u_{1} \operatorname{sn} u_{1} \operatorname{cn} u_{1}-\operatorname{dn} u_{2} \operatorname{sn} u_{2} \operatorname{cn} u_{2}\right]\right. \\
& \left.+2(1+\cos a)^{2}\left[\mathrm{E}\left(a, \operatorname{am} u_{1}\right)-\mathrm{E}\left(a \cdot \operatorname{am} u_{2}\right)\right]\right\} \\
& -\frac{\alpha}{3} \cos a .
\end{aligned}
$$

In the above-given formulas $u_{2}$ and $b$ are chosen in such a way that $-\mathrm{K}(a) \leqslant u_{2} \leqslant \mathrm{~K}(a)$, where $\mathrm{K}(a)$ is the complete elliptic integral of first kind and $\operatorname{sn} u$ and $\operatorname{cn} u$ are the Jacobian elliptic sine and cosine.

\section{LINEAR STABILITY ANALYSIS FOR EQUAL DISKS AND ZERO GRAVITY}

In order to perform the bifurcation analysis. we define the operator

$$
\begin{aligned}
& \mathcal{F}: \bar{X} \times \mathbb{R} \rightarrow C([-\Lambda, \Lambda] \times[0,2 \pi], \mathbb{R}) \times \mathbb{R}, \\
& \mathcal{F}:(f, a) \rightarrow\left(M(f)+a, \frac{1}{4 \pi \Lambda} \int_{-\Lambda}^{\Lambda} \int_{0}^{2 \pi} f^{2} d z d \theta\right),
\end{aligned}
$$

where $\bar{X}$ is the subset of functions of $C^{2}([-\Lambda, \Lambda]$ $\times[0,2 \pi], \mathbb{R}$ ) which fulfills boundary conditions (2) and (3). 
In these terms, the problem defined by expressions (2)(5) can be simply written as

$$
\mathcal{F}(F, P)=(0, v), \quad(F, P) \in \bar{Y} \times \mathbb{R} .
$$

The Frechet derivative of this operator at $(\bar{F}, \bar{P}) \in \bar{Y}$ $\times \mathbb{R}$ can be expressed as

$$
\begin{aligned}
\mathcal{F}^{\prime}(\bar{F}, \bar{P})(f, a)= & \left(M^{\prime}(\bar{F}) f+a, \frac{1}{2 \pi \Lambda} \int_{-\Lambda}^{\Lambda} \int_{0}^{2 \pi} \overline{F f} d z d \theta\right), \\
& (f, a) \in \overline{\bar{X}} \times \mathbb{R} .
\end{aligned}
$$

where, for axisymmetric $\bar{F}$. the Frechet derivative of $M(\bar{F})$ reads

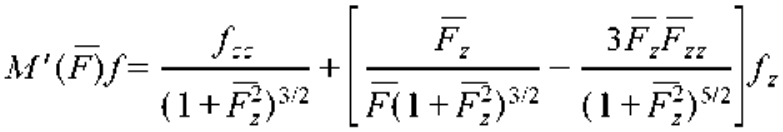

$$
\begin{aligned}
& +\frac{f+f_{\theta \theta}}{\overline{F^{2}}\left(\mathbf{I}+\bar{F}_{\varepsilon}^{2}\right)^{1 / 2}} \\
& =\frac{1}{\bar{F}} \frac{d}{d z}\left(\frac{\overline{F f_{z}}}{\left(1+\overline{\left.F_{z}^{2}\right)^{3 i 2}}\right.}\right)+\frac{f+f_{\theta \theta}}{\overline{F^{2}}\left(1+\bar{F}_{z}^{2}\right)^{1 / 2}} .
\end{aligned}
$$

A necessary condition for bifurcation in the neighborhood of an equilibrium solution, i.e., for the existence of more than one solution of the linearized problem obtained from (11), is the nonfulfilment of the implicit function theorem (Chow and Hale ${ }^{26}$ ). In such points, the Frechet deriva- tive is not one-to-one, or equivalently, its null space, $\mathcal{N}\left(\mathcal{F}^{\prime}(\bar{F}, \bar{P})\right)$, contains nonzero vectors. As stability character usually changes on bifurcation points, a practical method of determining possible stability limits is to find the parameter values for which the null space has other vectors than zero.

In Secs. III A and III B. the stability limits of axisymmetric equilibrium shapes due to axisymmetric and nonaxisymmetric perturbations. as well as the corresponding unstable modes. will be determined for the case $H=0$.

\section{A. Minimum volume stability limit (considering only axisymmetric perturbations)}

The perturbed problem to be solved in the neighborhood of any equilibrium solution of the boundary problem (I)-(4) is given by

$$
\begin{aligned}
& M^{\prime}(F) f+q=0, \\
& f( \pm \Lambda)=0, \quad \int_{-\Lambda}^{\Lambda} F f d z=0,
\end{aligned}
$$

with $f_{\theta}=f_{\theta \theta}=0$.

The function $F_{z}$, where $F$ is any axisymmetric equilibrium solution, is always a solution of Eq. (13) with $q=0$ since as $M(F)=-P$, then $(d / d z)[M(F)]=M^{\prime}(F) F_{z}=0$. Therefore, the general solution of Eq. (13) can be easily obtained using the constants variation teclunique. The solution can be then written in terms of elliptic variables as follows:

$$
\begin{aligned}
f(z(u))= & A \frac{\operatorname{sn} u \operatorname{cn} u}{\operatorname{dn}^{2} u+\cos a}+B \frac{\operatorname{dn} u\left(2 \operatorname{sn}^{2} u-1\right)+\operatorname{sn} u \operatorname{cn} u\left[\left(1+\cos ^{2} a\right) u-2 \mathrm{E}(a \cdot \operatorname{am} u)\right]}{\operatorname{dn}^{2} u+\cos a} \\
& +Q \frac{\operatorname{dn} u \operatorname{sn}^{2} u+\operatorname{sn} u \operatorname{cn} u[u-\mathrm{E}(a, \operatorname{am} u)]}{\operatorname{dn}^{2} u+\cos a}
\end{aligned}
$$

where $z(u)$ is given by the second relation of $(7)$ and

$$
Q=-\frac{\alpha(1+\cos a)^{2}}{2} q .
$$

The fulfillment of boundary conditions (14) leads to an homogeneous algebraic system of linear equations, which has a nontrivial solution if, and only if,

$$
\begin{aligned}
& 2 \sqrt{\left(1-\frac{1}{\alpha}\right)\left(\frac{1}{\alpha}-\cos ^{2} a\right)}\left[-\sqrt{\left(1-\frac{1}{\alpha}\right)\left(\frac{1}{\alpha}-\cos ^{2} a\right)}\right. \\
& \quad \times\left(-\frac{\cos ^{2} a}{2} \Delta u^{2}+\left(1+\cos ^{2} a\right) \Delta E \Delta u-\frac{3}{2} \Delta E^{2}+\frac{2}{\alpha}\right) \\
& +\frac{1}{\sqrt{\alpha}} \frac{u_{2}}{\left|u_{2}\right|}\left(\left(\frac{1}{\alpha}-2 \cos ^{2} a+\frac{\cos ^{2} a}{\alpha}\right) \Delta u\right. \\
& \left.\left.+2\left(1+\cos ^{2} a-\frac{2}{\alpha}\right) \Delta E\right)\right]=0 .
\end{aligned}
$$

where $\Delta u=u_{1}-u_{2}$ and $\Delta E=\mathrm{E}\left(a \cdot \operatorname{am} u_{1}\right)-\mathrm{E}\left(a, \operatorname{am} u_{2}\right)$.

The preceding expression defines the locus of bifurcation points [the points where $\mathcal{N}(\mathcal{F}(\bar{F}, \bar{P})) \neq\{(0,0)\}]$ in the parameters plane $(V, \Lambda)$. Such an expression coincides with the analytical expression of stability limits obtained by Gillette and Dyson ${ }^{2}$ making use of the conjugate point condition. In recent papers ${ }^{11,25}$ the same stability envelopes have been numerically obtained using alternate techniques. The solution of (16) is represented in Fig. 2 for the sake of completeness. To better understand the behavior of the stability limit we are going to study later, here we summarize some results already known, ${ }^{2,6,23,24}$

In general. Eq. (16) has three solutions: (a) $\alpha=1$, (b) $\alpha=1 / \cos ^{2} a$, (c) an intermediate value, $1<\alpha<1 / \cos ^{2} a$, which makes zero the second factor, included in brackets, on the left-hand side of expression (16).

Solutions $\alpha=1$ and $\alpha=1 / \cos ^{2} a$. In these cases, the practical stability limit is obtained for $\Delta u=2 \mathrm{~K}(a)$ and $\Delta \mathrm{E}$ 


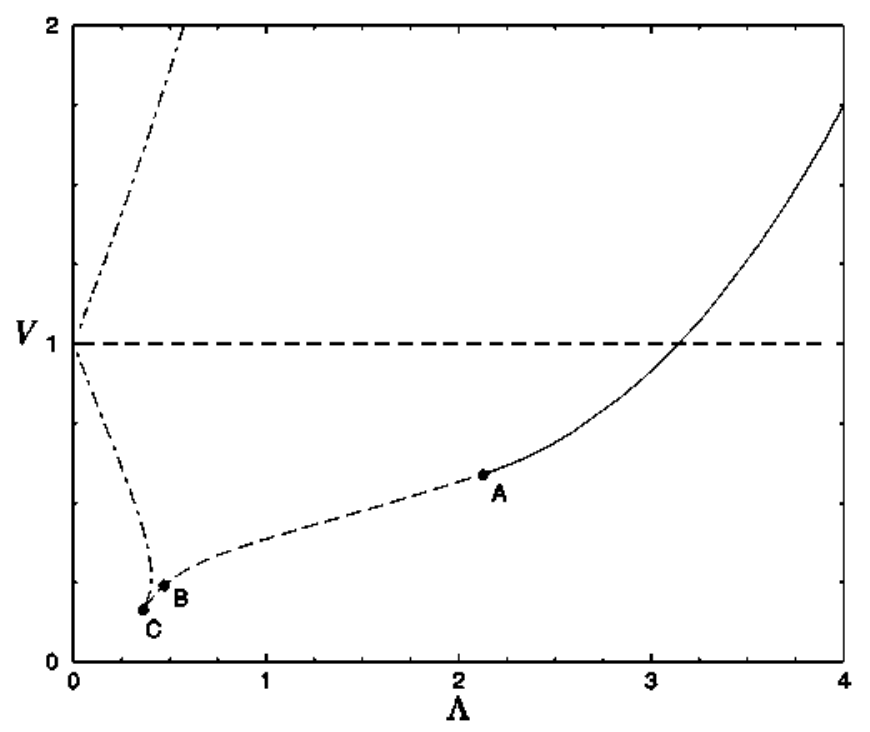

FIG. 2. Stability limits of axisymmetric liquid bridges. Solid line corresponds to axisymmetric breakages (nonsymmetric to the midplane and leading to unequal drops), dashed line to axisymmetric breakages (symmetric to the midplane and leading to equal drops), and dot-dashed lines to nonaxisymmetric, reversible perturbations.

$=2 \mathrm{E}(a)(0 \leqslant a \leqslant \pi / 2)$, when the equilibrium shape completes a period of the corresponding Plateau curve (unduloid). The solutions of the perturbed problem (13)-(14) are then proportional to $\left(F_{z}, 0\right)$.

Slenderness and volume ratio are given by

$$
\begin{aligned}
& \Lambda_{e}=\sqrt{\alpha}(\mathrm{K}(a) \cos a+\mathrm{E}(a)), \\
& v_{e}=\frac{\alpha}{3}\left[\frac{2(1+\cos a)^{2} \mathrm{E}(a)}{\mathrm{K}(a) \cos a+\mathrm{E}(a)}-\cos a\right],
\end{aligned}
$$

with the parameter $a$ ranging between 0 and $\pi / 2$ (see Fig. 2). In the above-given expressions, $\alpha=1$ and $\alpha=1 / \cos ^{2} a$ correspond to the stability limit in the regions $v<1$ and $v>1$, respectively (both are represented by the solid line in Fig. 2). However, for $\alpha=1$ the stability limit is of practical interest only when $0 \leqslant a \leqslant a_{1}$, where $a=a_{1}$ corresponds to the socalled "double-point" (point $A$ in Fig. 2) defined in the following. In Fig. 2 this curve has been plotted only up to this limit. although it further continues for smaller values of $\Lambda$ (see. e.g., Fig. 2 in Ref. 25).

Solution $\mathbf{I}<\alpha<1 / \cos ^{2} a$. The condition to have nontrivial solutions in this case is

$$
\begin{aligned}
& {\left[\sqrt{\left(1-\frac{1}{\alpha}\right)-\frac{u_{2}}{\left|u_{2}\right|} \sqrt{\alpha}} \sqrt{\left.\left(\frac{1}{\alpha}-\cos ^{2} a\right)\left(\frac{\Delta u}{2}-\Delta E\right)\right]}\right.} \\
& \quad \times\left[\sqrt{\left(\frac{1}{\alpha}-\cos ^{2} a\right)}+\frac{u_{2}}{\left|u_{2}\right|} \sqrt{\alpha} \sqrt{\left(1-\frac{1}{\alpha}\right)}\right. \\
& \left.\quad \times\left(\cos ^{2} a \frac{\Delta u}{2}-\Delta E\right)\right] \\
& =-\frac{\alpha}{4} \sqrt{\left(1-\frac{1}{\alpha}\right)\left(\frac{1}{\alpha}-\cos ^{2} a\right) \Delta E^{2} .}
\end{aligned}
$$

with

$$
\begin{aligned}
& \Delta u=2 n \mathrm{~K}(a)-2 u_{2}, \\
& \Delta E=2 n \mathrm{E}(a)-2 \mathrm{E}\left(a, \operatorname{am} u_{2}\right), \quad \frac{1}{\sqrt{\alpha}}=\operatorname{dn} u_{2} .
\end{aligned}
$$

The corresponding slenderness and volume ratio are

$$
\begin{aligned}
& \Lambda_{e}(a)=\frac{\sqrt{\alpha}}{2}[\Delta u \cos a+\Delta E] . \\
& v_{e}(a)=\alpha\left[\frac{2}{3} \frac{\left.(1+\cos a)^{2} \Delta E-\frac{1}{\sqrt{\alpha}} \sqrt{\left(1-\frac{1}{\alpha}\right)\left(\frac{1}{\alpha}-\cos ^{2} a\right) \frac{u_{2}}{\left|u_{2}\right|}}-\frac{\cos a}{3}\right] .}{(\Delta u \cos a+\Delta E)} .\right.
\end{aligned}
$$

On the one hand. when $0 \leq a<\pi / 2$ (the meridian shape of the interface corresponds to an unduloid). the first mode instability is obtained for $n=\left(1+u_{2} /\left|u_{2}\right|\right) / 2$ [recall that $u_{2}$ was chosen in the range $\left.-\mathrm{K}(a)<u_{2}<\mathrm{K}(a)\right]$. On the other hand, if $\pi / 2<a \leqslant \pi$ (the interface contour is a piece of nodoid), expressions (18) and (19) are applicable only for $n=\left(-1+u_{2} /\left|u_{2}\right|\right) / 2$. Otherwise. the interface shape presents unbounded derivatives and/or unrealistic double points where the interface intersects itself. For $n=0$ the curve (19) is in the region $v>1$, where it is well known that the practical minimum volume stability limit is given by (17) with $\alpha=1 / \cos ^{2} a$ (Refs. 6 and 23). Therefore, the curve (19) is of practical interest for either $0 \leq a<\pi / 2$ and $u_{2}>0$. or $\pi / 2$ $<a \leqslant \pi$ and $u_{2}<0$, i.e., $n=u_{2} /\left|u_{2}\right|=\cos \alpha /|\cos a|$. Actually. (18) and (19) describe the practical stability limit between points $A$ and $C$ (the dashed line in Fig. 2). At point $A$ the curves (17) for $\alpha=1$ and (19) for $\cos a>0\left(u_{2}>0\right.$ and $n$ $=1$ ) have a first-order contact. Analytically, point $A$ is determined by the condition

$$
\mathrm{K}\left(a_{1}\right)=2 \mathrm{E}\left(a_{1}\right),
$$

which has the solution $a_{1}=1.14065 \ldots$ corresponding to $\Lambda=\Lambda_{A}=2.127 \ldots$ and $v=0.591 \ldots$ (see Fig. 2). 
Point $C$ corresponds to the first point of curve (19) in which the interface shape presents an unbounded derivative $F$ : [given by (15) for $A=-\sin ^{2} a$ and $B=Q=0$ ]. Such a condition corresponds to $a=a_{2}$ such that $\mathrm{dn}^{2} u_{2}=-\cos a_{2}$, which implies that $a_{2}=1.616196 \ldots, \Lambda_{C}=0.361131 \ldots$ and $v_{C}=0.164315 \ldots$.

The so-called catenoidal limit (point $B$ in Fig. 2) is obtained for $a=\pi / 2$. In this case $u_{2}$ and $\alpha$ tend to infinity, but $\Delta u$ remains bounded and fulfills

$$
\begin{aligned}
(1+ & \left.3 \tanh ^{2}\left(\frac{\Delta u}{2}\right)\right) \tanh \left(\frac{\Delta u}{2}\right)-\frac{\Delta u}{2}\left(1-3 \tanh ^{2}\left(\frac{\Delta u}{2}\right)\right) \\
& \times\left(1-2 \tanh ^{2}\left(\frac{\Delta u}{2}\right)\right)+3\left(\frac{\Delta u}{2}\right)^{2} \tanh \left(\frac{\Delta u}{2}\right) \\
& \times\left(1-\tanh ^{2}\left(\frac{\Delta u}{2}\right)\right)^{2}=0 .
\end{aligned}
$$

The previous expression results after taking limits in (18) for $\cos a$ tending to zero. As a consequence of that, point $B$ corresponds to $\Delta u=4.47836 \ldots, \Lambda_{B}=0.47179 \ldots$, and $v_{B}=0.24048 \ldots$.

The solutions of the perturbed problem (13)-(14) corresponding to the stability limits analyzed in this region ( 1 $\left.<\alpha<1 / \cos ^{2} a\right)$ are proportional to $\left(f_{2}, q_{2}\right)$, where

$$
\begin{aligned}
q_{2}= & \frac{3 \Delta E}{\alpha(1+\cos a)}-\frac{2}{\alpha^{2}(1+\cos a)^{3}} \\
& \times\left(\Delta E-\frac{\left(1+\cos ^{2} a\right)}{2} \Delta u\right)-\frac{12 v \Lambda}{\alpha^{5 / 2}(1+\cos a)^{3}} .
\end{aligned}
$$

and $f_{2}$ is given by expression (15) with

$$
\begin{aligned}
A= & \sin ^{2} a\left[\frac{3}{2}(\mathrm{~K}(a) \cos a+\mathrm{E}(a)) \Delta E+\frac{1}{2 \alpha}(\mathrm{K}(a) \Delta E\right. \\
& \left.-\mathrm{E}(a) \Delta u)-\frac{3 v \Lambda}{\alpha^{3 / 2}} \mathrm{~K}(a)\right] \\
B= & \frac{3}{2}(1+\cos a) \Delta E+\frac{1}{2 \alpha}(\Delta E-\Delta u)-\frac{3 v \Lambda}{\alpha^{3 / 2}} . \\
Q= & -\frac{\alpha(1+\cos a)^{3}}{2} q_{2} .
\end{aligned}
$$

\section{B. Stability limit considering nonaxisymmetric perturbations}

Nonaxisymmetric perturbations must satisfy

$$
M^{\prime}(F) f+q=0, \quad \int_{-\Lambda}^{\Lambda} d z \int_{0}^{2 \pi} F f d \theta=0
$$

where $M^{\prime}(F)$ is given by (12), and the boundary and periodicity conditions

$$
\begin{array}{ll}
f( \pm \Lambda, \theta)=0, & f(z, \theta)=f(z, \theta+2 \pi) . \\
z \in[-\Lambda, \Lambda], & \theta \in[0,2 \pi[.
\end{array}
$$

Using the technique of variables separation, we obtain that the system (23)-(24) has no nontrivial nonaxisymmetric solution for $F_{z}$ bounded in $[-\Lambda, \Lambda]$. To slow that, the lead- ing equation of (23) must be multiplied by $F(z) a_{n}(z) \cos n \theta$. where $a_{n}(z)$ is the $n$th cosinus coefficient in the Fourier series of $f$. Then both members of the resulting equation must be integrated in $(z, \theta) \in]-\Lambda, \Lambda[\times] 0.2 \pi[$. After one integration by parts in the $z$ variable and taking into account that $a_{n}( \pm \Lambda)=0$ [see the first condition of (24)], we have

$$
\int_{-\Lambda}^{\Lambda}\left[\frac{F}{\left(1+F_{z}^{2}\right)^{3 / 2}}\left(\frac{d a_{n}}{d z}\right)^{2}+\frac{n^{2}-1}{F\left(1+F_{z}^{2}\right)^{1 / 2}} a_{n}^{2}\right] d z=0 .
$$

Now, if $n>0$, the identity $a_{n}(z) \equiv 0$ in $[-\Lambda, \Lambda]$ readily follows. In a similar way it is demonstrated that the $n$th sinus coefficient is zero for every solution of $(23)-(24)$. Therefore, as long as the value of $F_{z}$ is bounded in $[-\Lambda, \Lambda]$, only the previously analyzed bifurcations to axisymmetric modes can arise.

However, for $H=0$ an interesting case of bifurcation to stationary solutions appears in the axisymmetric equilibrium solution in which $F_{z} \rightarrow \pm \infty$ at the ends of the equilibrium shape $(z= \pm \Lambda)$. To deal with these solutions, the system (2) -(5) must be reformulated to allow unbounded $z$ derivatives in both the equilibrium and the perturbed solutions.

Let $(F=\bar{F}(t), z=\bar{Z}(t), \theta=\theta)$ be an axisymmetric solution in parametric form [for instance those ones given by expressions (7) taking $t=u$ ], which is now perturbed as follows:

$$
F=\bar{F}(t)+\frac{1}{1+\overline{F_{z}^{2}}} h(t, \theta), \quad z=\bar{Z}(t)-\frac{\overline{F_{z}}}{1+\overline{F_{z}^{2}}} h(t, \theta) .
$$

In these expressions, perturbations are measured along the normal vector to the axisymmetric equilibrium solution.

Using an explicit representation for the equilibrium shape $(t=z)$, in a first approximation the perturbation magnitude, $h=h(z, \theta)$, is obtained as solution of the following linearized problem:

$$
\begin{aligned}
& M^{\prime}(\bar{F}) h+q=0 . \quad \int_{0}^{2 \pi}\left(\int_{-\Lambda}^{\Lambda} \overline{F h} d z\right) d \theta=0, \\
& \lim _{z \rightarrow \pm \Lambda} \frac{h(z, \theta)}{\sqrt{1+\overline{F_{z}^{2}}}}=0, \quad h(z, \theta)=h(z, \theta+2 \pi), \\
& \theta \in[0.2 \pi[
\end{aligned}
$$

When $\bar{F}$ is bounded at either $z=-\Lambda$ or $z=\Lambda$, all terms in the Fourier series of the nonaxisymmetric solutions of (26) have zero coefficients, as can be easily seen using (25) and the boundary conditions of (26). However, when $\bar{F}_{z}$ is an unbounded function at both ends, the nontrivial nonaxisymmetric solution

$$
h_{1}(z, \theta)=A \cos \theta .
$$

fulfills the boundary conditions (26) [the solutions which are proportional to $\sin \theta$ are also considered in (27) by means of a variable change in $\theta$ ]. This nonaxisymmetric perturbation mode (27) has been previously reported by Slobozhanin et al. ${ }^{1.11}$ 
Therefore, when $H=0$, new curves of bifurcation can be found in the plane $(\Lambda, v)$ for $F_{z}=F_{t} / z_{u}$ unbounded at both ends. These curves are determined by the condition $z_{t}$ $=\left.\sqrt{\alpha}\left(\cos a+\operatorname{dn}^{2} u\right)\right|_{*_{1}, u_{2}}=0$. which implies $\operatorname{dn} u_{1}=\operatorname{dn} u_{2}$ $=\sqrt{-\cos a}$ and $\alpha=-1 / \cos a(\pi / 2 \leqslant a \leqslant \pi)$. The resulting stability limits (the dot-dashed lines of Fig. 2) are described in parametric form as

$$
\begin{aligned}
\Lambda_{e}(a)= & \frac{1}{2 \sqrt{-\cos a}}[1+\cos a \pm(\cos a \mathrm{~K}(a)+\mathrm{E}(a))] . \\
v_{e}(a)= & \frac{-2}{3 \cos a}\left(1+\cos ^{2} a+\frac{3 \cos a}{2}\right) \\
& +\frac{(1+\cos a)}{3 \Lambda_{e} \sqrt{-\cos a}}[1 \pm(1+\cos a) \mathrm{K}(a)] .
\end{aligned}
$$

These expressions correspond, respectively to the leftmost branch of the minimum volume stability limit curve (taking in the expressions the sign -) and to the maximum volume stability limit curve (taking the sign +) (see Refs. 11 and 25). When the minus sign is taken in these expressions. the resulting curve is in the region $v<1$ and intersects the curve given by (19) in point $C\left(a_{2}=1.616196 \ldots\right)$ determined in the previous section.

\section{BIFURCATION ANALYSIS IN THE NEIGHBORHOOD OF THE MINIMUM VOLUME STABILITY LIMIT FOR EQUAL DISKS AND ZERO GRAVITY}

The Lyapunov-Schmidt method is going to be used in the bifurcation analysis (Myshkis et al. ${ }^{1}$ Chow and $\mathrm{Hale}^{26}$ ). The problem to be solved in the axisymmetric case is

$$
\mathcal{F}(F . P)+(f . q) \Psi=\left(0, v+V^{\prime}\right) . \quad F( \pm \Lambda)=\mathbf{I} \pm H .
$$

whose solutions are expanded as $(F, P)=(\bar{F}+\epsilon f+u, \bar{P}$ $+\epsilon q+r)$. The pair $(\bar{F}, \bar{P})$ is the unperturbed solution of problem (11) where bifurcation takes place. However, perturbations are divided into two linearly independent components, $(f, q) \in \mathcal{N}\left(\mathcal{F}^{\prime}(\bar{F}, \bar{P})\right)$ and $(u, r)$, i.e.,

$$
\mathcal{F}^{\prime}(\bar{F}, \bar{P})(f, q)=\left(M^{\prime}(\bar{F}) f+q, \frac{1}{\Lambda} \int_{-\Lambda}^{\Lambda} \overline{F f} d z\right)=(0,0) .
$$

In fact, the parameter $\epsilon$, which stands for the magnitude of the interface deformation, is defined in such a way that the orthogonality condition $\langle(f, q),(u, r)\rangle=0$ holds. Here the inner product

$$
\langle(f, q),(h, s)\rangle=\frac{1}{\Lambda} \int_{-\Lambda}^{\Lambda} \bar{F} f h d z+s q,
$$

has been defined for all $(f, q)$ and $(h, s) \in C^{2}([-\Lambda, \Lambda], \mathbb{R})$ $\times \mathbb{R}$. Such an orthogonality condition uniquely defines $\epsilon$ as

$$
\epsilon=\frac{\langle(F-\bar{F} \cdot P-\bar{P}),(f, q)\rangle}{\langle(f, q),(f, q)\rangle} .
$$

In the boundary value problem (28) [see also Eq. (1)], $V$, $H, B$, and $W$ appear as small parameters to be used to study the bifurcation in the neighborhood of the solution $(\bar{F}, \bar{P})$ which corresponds to volume $v$ (of order unity) and fulfills the requirement $\mathcal{N}\left(\mathcal{F}^{\prime}(\bar{F}, \bar{P})\right) \neq\{(0,0)\}$

According to the Lyapunov-Schmidt method, in the neighborhood of the bifurcation point corresponding to $\epsilon$ $=V=H=B=W=0,(u, r)=(0,0)$. problem (28) uniquely defines the functions $u(z ; \epsilon, \mathcal{V}, H, B, W), r(\epsilon, V, H, B, W)$. and $\Psi(\epsilon . V, H . B . W)$, which can be expanded as

$$
\begin{aligned}
u(z ; \epsilon, Y, H, B, W)= & \sum_{i=2}^{5} \epsilon_{i} u_{i}(z)+\sum_{i=1}^{5} \sum_{j=1}^{5} \epsilon_{i} \epsilon_{j} u_{i j}(z) \\
& +\sum_{i=1}^{5} \sum_{j=1}^{5} \sum_{k=1}^{5} \epsilon_{i} \epsilon_{j} \epsilon_{k} u_{i j k}(z)+\cdots \\
r(\epsilon, V, H, B, W)= & \sum_{i=2}^{5} \epsilon_{i} r_{i}+\sum_{i=1}^{5} \sum_{j=1}^{5} \epsilon_{i} \epsilon_{j} r_{i j} \\
& +\sum_{i=1}^{5} \sum_{j=1}^{5} \sum_{k=1}^{5} \epsilon_{i} \epsilon_{j} \epsilon_{k} r_{i j k}+\cdots, \\
\Psi(\epsilon, V, H, B, W)= & \sum_{i=1}^{5} \epsilon_{i} g_{i}+\sum_{i=1}^{5} \sum_{j=1}^{5} \epsilon_{i} \epsilon_{j} g_{i j} \\
& +\sum_{i=1}^{5} \sum_{j=1}^{5} \sum_{k=1}^{5} \epsilon_{i} \epsilon_{j} \epsilon_{k} g_{i j k}+\cdots,
\end{aligned}
$$

where the notation $\epsilon_{1}=\epsilon: \epsilon_{2}=V: \epsilon_{3}=H ; \epsilon_{4}=B ; \epsilon_{5}=W$ has been used. The different coefficients in the expansions $u_{i j}, u_{i j k}, r_{i j}, r_{i j k}, g_{i j}, g_{i j k} \ldots \ldots$ are symmetric to index permutations.

The bifurcation equation will be

$$
\Psi(\epsilon, \mathrm{V}, H, B, W)=0 \text {. }
$$

Notice that setting $\Psi=0$ the problem defined by (28) coincides with the problem given by (1)-(4).

Before solving the problem (28) it is convenient to analyze the symmetries involved in the problem, which allow us to anticipate some characteristics of the bifurcation equation (32) and to ease the algebra involved (see, e.g., Meseguer et al. ${ }^{7}$ Vega and Perales, ${ }^{21}$ and Perales ${ }^{27}$ ). When the vector of the null space $\mathcal{N}\left(\mathcal{F}^{\prime}(\bar{F}, \bar{P})\right)$ is proportional to $(f, q)$ $=\left(\bar{F}_{z}, 0\right)$ [as is the case along the curve (17). the solid line in Fig. 2], problem (28) remains invariant under the following set of symmetries (notice that $\bar{F}_{z}$ is now an odd function of $z$ ):

$$
z \rightarrow-z, \epsilon--\epsilon, H \rightarrow-H, B \rightarrow-B, \Psi \rightarrow-\Psi .
$$

From these symmetries the following identity

$$
\Psi(\epsilon, \mathcal{V}, H, B, W)=-\Psi\left(-\epsilon, V^{\prime},-H_{-}-B, W\right)
$$

can be easily deduced.

Thus, without any further calculation, it can be inferred that the only nonzero first-order coefficients are those corresponding to the terms in $H$ and $B$. Moreover, the secondorder and third-order coefficients to be taken into account are 
those proportional to $\epsilon V, \epsilon W$, and $\epsilon^{3}$. This analysis leads to the following (simplified) bifurcation equation where only nonzero relevant terms have been included:

$$
g_{3} H+g_{4} B+2 g_{12} \epsilon V^{\prime}+2 g_{15} \epsilon W+g_{111} \epsilon^{3}+\cdots=0,
$$

which represents a branching to unstable axisymmetric solutions if $H=B=0$ and breaks into two branches otherwise.

In the cases corresponding to $(f, q)=\left(f_{2}, q_{2}\right)$ [such as those described by (19), the dashed line in Fig. 2], problem (28) remains invariant under the following set of symmetries [notice that now $f_{2}(z)=f_{2}(-z)$ ]:

$$
z \rightarrow-z . H \rightarrow-H . B \rightarrow-B .
$$

Thus, the identity

$$
\Psi\left(\epsilon, V^{\prime}, H, B, W\right)=\Psi(\epsilon, Y,-H,-B, W)
$$

holds and. as a result the only first-order coefficients which are nonzero are those corresponding to the terms in $V$ and $W$. Among the second-order coefficients the only ones to be taken into account are those corresponding to the terms in $B^{2}, H^{2}$, and $B H$. All the third-order coefficients are irrelevant when are compared with the previous ones.

Therefore, the (simplified) bifurcation equation reduces to

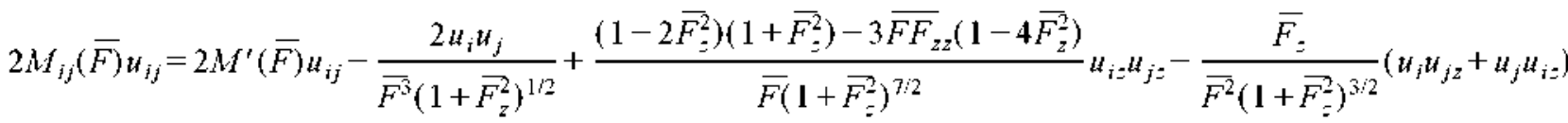

$$
\begin{aligned}
& -\frac{3 \bar{F}_{z}}{\left(1+\bar{F}_{z}^{2}\right)^{5 i 2}}\left(u_{i z} u_{j z z}+u_{j z} u_{i z z}\right) \\
& M_{u n}(\bar{F}) u_{u i}=M^{\prime}(\bar{F}) u_{u i}-\frac{2 u_{\imath} u_{i j}}{\overline{F^{3}}\left(1+\overline{F_{z}^{2}}\right)^{1 / 2}}+\frac{u_{i}^{3}}{\bar{F}^{4}\left(1+\bar{F}_{z}^{3}\right)^{1 / 2}}-\frac{\bar{F}_{z} u_{i j} u_{i z}}{\bar{F}^{2}\left(1+\bar{F}_{z}^{2}\right)^{3 / 2}}+\frac{\overline{F_{z}} u_{i}^{2} u_{i z}}{\overline{F^{3}}\left(1+\bar{F}_{z}^{2}\right)^{3 / 2}}-\frac{\left(1-2 \bar{F}_{z}^{2}\right) u_{i} u_{i z}^{2}}{2 \overline{F^{2}}\left(1+\bar{F}_{z}^{3}\right)^{5 / 2}}
\end{aligned}
$$

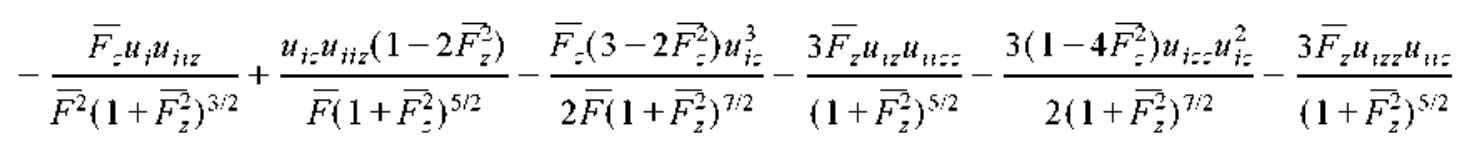

$$
\begin{aligned}
& -\frac{3 \bar{F}_{z z}\left(1-4 \bar{F}_{z}^{3}\right) u_{i z} u_{i z}}{\left(1+\overline{F_{z}^{2}}\right)^{7 / 2}}+\frac{5 \bar{F}_{z} \bar{F}_{z z}\left(3-4 \bar{F}_{z}^{3}\right) u_{i z}^{3}}{2\left(1+\overline{F_{z}^{2}}\right)^{9 / 2}}
\end{aligned}
$$

Expressions of $M(\bar{F})$ and $M^{\prime}(\bar{F}) u_{i}$ are given by $(5)$ and (12) (with $u_{i \theta \theta}=0$ ), respectively, whereas $M_{i j}(\bar{F}) u_{i j}$ and $M_{i j i}(\bar{F}) u_{i j}$ are given by

which represents a turning point.

Introducing the expressions $F=\bar{F}+\epsilon f+u, P=\bar{P}+\epsilon q$ volume stability limit considered in Sec. III A: (1) $\Delta>\Delta$ with $f=\bar{F}_{z}$ and $q=0 ;$ (2) $\Lambda<\Lambda_{A}$ with $f=f_{2}$ given by (15) and (22), and $q_{2} \neq 0$. given by (2I).

To obtain the formulation of these problems the mean

$$
\begin{aligned}
M(F)= & M(\bar{F})+\sum_{i=1}^{5} \epsilon_{i} M^{\prime}(\bar{F}) u_{j}+\sum_{i=1}^{5} \sum_{j=1}^{5} \epsilon_{i} \epsilon_{j} M_{i j}(\bar{F}) u_{i j} \\
& +\sum_{i=1}^{5} \sum_{j=1}^{5} \sum_{k=1}^{5} \epsilon_{i} \epsilon_{j} \epsilon_{k} M_{i j k}(\bar{F}) u_{i j k}+\cdots .
\end{aligned}
$$


TABLE I. Coefficients of the bifurcation equation in the range $\Lambda>\Lambda_{A}$,

\begin{tabular}{lr}
\hline \hline$\Lambda_{A}<\Lambda<\pi(\alpha=1)$ or $\Lambda>\pi\left(\alpha=1 / \cos ^{2} a\right)$ & $\Lambda=\pi$ \\
\hline$g_{111}=\alpha^{-1 / 2} \frac{2}{\pi(1-\alpha \cos a)^{2}}\left[\frac{(\mathrm{K}(a)-\alpha \mathrm{E}(a))^{2}}{\mathrm{~K}(a)-2 \alpha \mathrm{E}(a)}-\left(1+\alpha^{2} \cos ^{2} a\right) \mathrm{K}(a)+2 \alpha \mathrm{E}(a)\right]$ & $g_{111} \cong-\frac{3}{2}$ \\
$g_{12}=-\frac{\alpha^{1 / 2}}{\mathrm{~K}(a)-2 \alpha \mathrm{E}(a)} \frac{4(\mathrm{~K}(a) \cos a+\mathrm{E}(a))(\mathrm{K}(a)-\alpha \mathrm{E}(a))}{\pi(1-\alpha \cos a)(1+\alpha \cos a)^{2}}$ & $g_{12} \cong 1$ \\
$g_{15}=\alpha^{-1 / 2} \frac{1+\alpha \cos a}{6 \pi(1-\alpha \cos a)}\left[(\mathrm{K}(a)-\alpha \mathrm{E}(a))\left[3+4 \alpha^{2} \cos ^{2} a+\frac{\alpha \mathrm{E}(a)}{\mathrm{K}(a)-2 \alpha \mathrm{E}(a)}\right]\right.$ & $g_{15} \cong-1$ \\
$g_{3}=-\frac{4}{\pi} \frac{1}{1+\alpha \cos a}$ & $g_{3} \cong-\frac{2}{\pi}$ \\
$g_{4}=\frac{2}{\pi} \frac{\mathrm{K}(a) \cos a+\mathrm{E}(a)}{(1-\cos a)}|1-v|$. & $g_{4} \cong 2$ \\
$v=\frac{\alpha}{3}\left[\frac{2(1+\cos a)^{2} \mathrm{E}(a)}{\mathrm{K}(a) \cos a+\mathrm{E}(a)}-\cos a\right]$ & \\
\hline \hline
\end{tabular}

$M_{i j}(\bar{F}) u_{i j}+r_{i j}+g_{i j} f=-\Delta_{i, 1} \Delta_{j .5} \overline{F f}$

$\frac{1}{\Lambda} \int_{-\Lambda}^{\Lambda}\left(2 \bar{F} u_{i j}+u_{i} u_{j}\right) d z+g_{i j} q=0$,

$$
u_{i j}( \pm \Lambda)=0, \quad \frac{1}{\Lambda} \int_{-\Lambda}^{\Lambda} \bar{F}_{u_{i j}} f d z+r_{i j} q=0 .
$$

The symmetries analysis indicates that $g_{11}=g_{13}=g_{14}=0$ in the case $f=\bar{F}_{z}$ and $g_{13}=g_{14}=0$ for $f=f_{2}$. Moreover, provided that quantities $g_{3}$ and $g_{4}$ are nonzero. the coefficients $g_{33}, g_{44}$, and $g_{34}$ are nonrelevant when $f=\bar{F}_{z}$. Similarly, when $f=f_{2}$, owing to the fact that $g_{2}, g_{5}$, and $g_{11}$ are not zero, the coefficients $g_{12}$ and $g_{15}$ are not needed

Order $\epsilon_{1}^{3}\left(\epsilon^{3}\right)$ :

$$
\begin{aligned}
& M_{111}(\bar{F}) u_{111}+r_{111}+g_{111} f=0, \\
& \frac{1}{\Lambda} \int_{-\Lambda}^{\Lambda}\left(\bar{F} u_{111}+f u_{11}\right) d z+g_{111} q=0, \\
& u_{111}( \pm \Lambda)=0, \quad \frac{1}{\Lambda} \int_{-\Lambda}^{\Lambda} \bar{F} u_{111} f d z+r_{111} q=0 .
\end{aligned}
$$

(this problem is nonrelevant if $f=f_{2}$ ).

Using the Frechet derivative operator (29), all the abovedescribed problems take the form

$$
\mathcal{F}^{\prime}(\bar{F}, \bar{P})(u, r)+g(f, q)+(h, a)=(0,0)
$$

where $g$ is the corresponding coefficient of the bifurcation equation and the vector $(h, a) \in C([-\Lambda, \Lambda], \mathbb{R}) \times \mathbb{R}$ only depends on the solutions of lower order problems. The inner product $(30)$ of the preceding equation and $(f, q)$ $\in \mathcal{N}\left(\mathcal{F}^{\prime}(\bar{F}, \bar{P})\right)$ give the resolubility condition

$$
g=-\frac{\langle(h, a) \cdot(f, q)\rangle}{\langle(f, q),(f, q)\rangle}
$$

because $\quad\left\langle\mathcal{F}^{\prime}(\bar{F}, \bar{P})(u, r),(f, q)\right\rangle=\left\langle(u, r), \mathcal{F}^{\prime}(\bar{F}, \bar{P})(f, q)\right\rangle$ $=0$. as can be easily proven. Therefore. to calculate the relevant coefficients of the bifurcation equation the higher order problems are not needed to be fully solved.

\section{A. Bifurcation in the range $\boldsymbol{\Lambda}>\boldsymbol{\Lambda}_{\boldsymbol{A}}$}

The possible bifurcation points are the stability limits given by the parametric expressions (17) (the solid line in Fig. 2) for $\alpha=1 / \cos ^{2} \alpha(0 \leqslant a \leqslant \pi / 2)$ and $\alpha=\mathrm{I}\left(0 \leqslant a \leqslant a_{1}\right)$. Here the null space of $\mathcal{F}^{\prime}(\bar{F}, \bar{P})$ is generated by the vector $(f . q)=\left(\overline{F_{z}}, 0\right)$.

The solutions of the different order problems yield the expressions of the relevant coefficients and functions of the asymptotic expansions of $u(z), r$, and $\Psi$. The obtained results are collected and summarized in Appendix 1. Finally, the following bifurcation equation, which is valid in the range of values $\Lambda>\Lambda_{A}=2.127 \ldots$, results

$$
\begin{aligned}
& \frac{1-\alpha \cos a}{(1+\alpha \cos a)(1-\sqrt{\alpha \cos a})^{2}}\left[\widetilde{\epsilon}^{3} g_{111}+\widetilde{\epsilon} V g_{12}+\widetilde{\epsilon} W g_{15}\right. \\
& \left.+H g_{3}+B g_{4}+\cdots\right]=0 .
\end{aligned}
$$

The parameter $\tilde{\epsilon}$ is defined as

$$
\widetilde{\boldsymbol{\epsilon}}=\frac{\boldsymbol{\epsilon}(\mathbf{I}-\alpha \cos a)}{(1+\alpha \cos a)} \text {. }
$$

The analytical expressions of the coefficients of the previous bifurcation equation as well as their limit values for $\Lambda=\pi$, are shown in Table I and plotted in Fig. 3. Notice that the corresponding limit values for $\Lambda=\pi$ (the cylindrical configuration) coincide with the data previously published (Meseguer et al. ${ }^{8}$ Vega and Perales ${ }^{22}$ ). Moreover, in the neighborhood of the "double point" $\left(\Lambda=\Lambda_{A}\right)$ the coefficients $g_{111}, g_{12}$, and $g_{15}$ tend to some nonfinite value, as can be easily seen from their analytical expressions because 


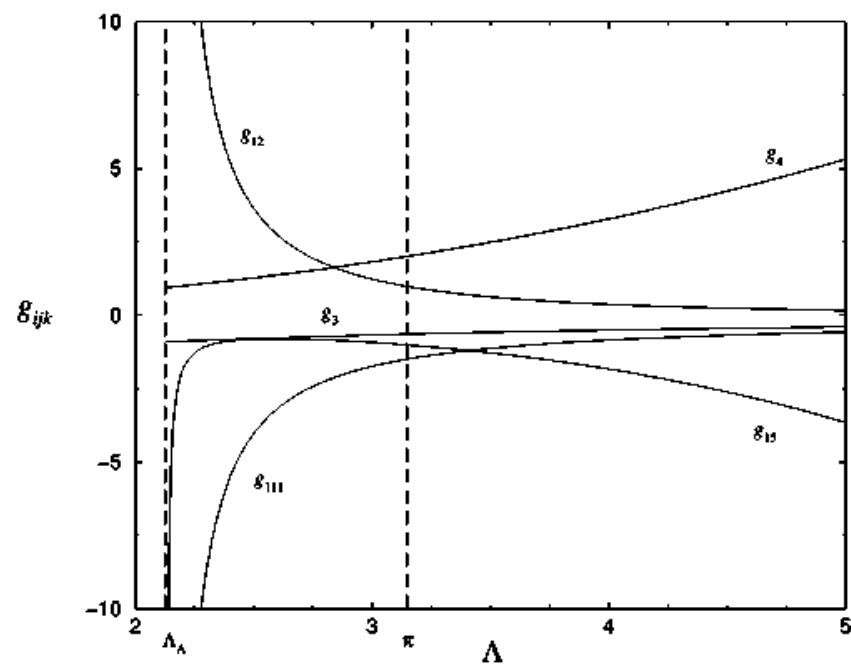

FIG. 3. Bifurcation equation coefficients as a function of slenderness, $\Lambda$ $>\Lambda_{A}$.

$\mathrm{K}(a)$ equals $2 \mathrm{E}(a)$ at this point. However. the relationships $g_{111} / g_{12}$ and $g_{15} / g_{12}$ remain finite while $g_{3} / g_{12}$ and $g_{4} / g_{12}$ vanish.

Equation (37) implies that for small values of $B$ and $H$ the stability limit is shifted in an amount proportional to two-thirds power of quantity $g_{3} H+g_{4} B$. This shift can be zero for particular combinations of $H$ and $B(H / B$ $=-g_{4} / g_{3}$ ), which are represented in Fig. 4. In such a case, the symmetry of the problem, broken by any of the two imperfections, is recovered and the change in the stability limit is determined by the nonconsidered high order terms. In fact, the symmetries analysis implies that the terms which should be considered are those of order $V^{\prime} H, V^{\prime} B, \epsilon^{2} H, \epsilon^{2} B$, $\epsilon l^{2}$, and $\epsilon^{5}$.

As has been previously mentioned, when $H=B=W$ $=0$, Eq. (37) leads to subcritical bifurcation of pitclfork type near the marginally stable equilibrium solutions corresponding to constant slenderness values with $\Lambda>\Lambda_{A}$. In such a

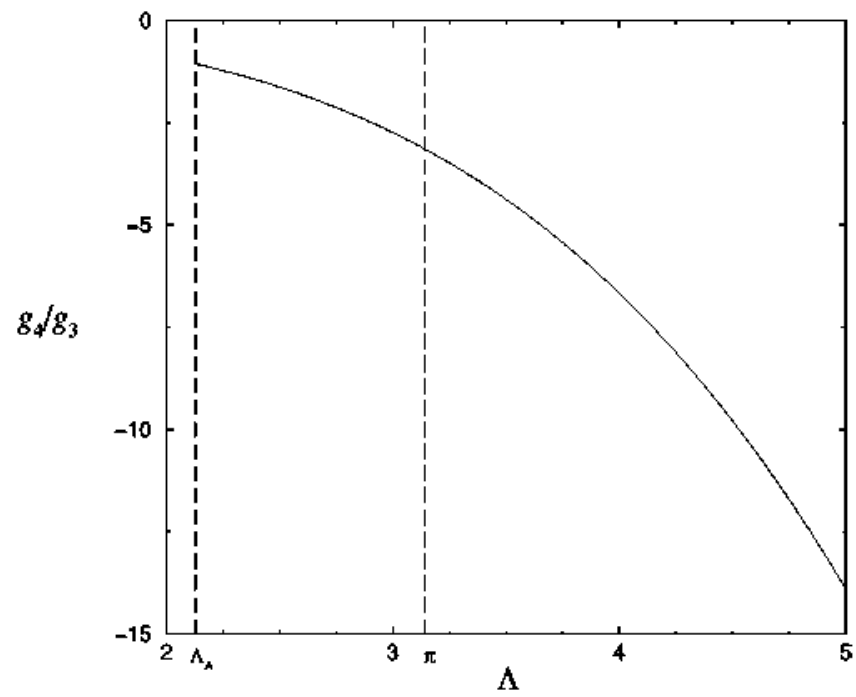

FIG. 4. Ratio $H: B$ for recovering a pitchfork bifurcation as a function of slenderness. case, the bifurcated branches of equilibrium solutions are in the stable range $\left(v \geqslant v_{e}\right)$ of the bifurcation diagram $(\epsilon, v)$. This behavior is observed on the curve corresponding to the values $H=B=W=0$ of Fig. 5, where the bifurcation diagram in the $\left(R_{1 / 4}, v\right)$ plane for $\Lambda=4$ is shown. Parameter $R_{1 / 4}$ is defined as the equilibrium shape radius at $z=-\Lambda / 2$ and it is approximated by $R_{1 / 4}=\bar{F}(-\Lambda / 2)+\epsilon \bar{F}_{z}(-\Lambda / 2)$.

Small imperfections, such as axial gravity or disks diameter inequality. leading to antisymmetric perturbations with respect to $z=0$ break the diagram symmetry [see. e.g., Figs. $5(\mathrm{a})-5(\mathrm{c})]$. This symmetry breaking transforms the original bifurcation point (of pitchfork type for $H=B=W=0$ ) into a turning point and an additional isolated branch. In Fig. 5(c). the bifurcation diagram obtained for both $H \neq 0$ and $B \neq 0$ is shown. A small inequality of the disks diameter partially cancels the effect of a small positive axial gravity, shifting the turning point to a value of $\mathrm{V}_{\mathrm{c}}=\mathrm{J}_{c 1 \mathrm{c}}$ smaller than that corresponding to equal disks and the same value of $B\left[\mathrm{Y}_{C 1 a}\right.$ in Fig. 5(a)]. If only negative values of the Bond number are considered, the result is equivalent to adding both of these instabilizing effects and the turning point is moved toward a value, $V_{c 2_{c}}$, greater than the critical volume corresponding to $H=0\left(V_{c \mid a}\right)$.

The effect of an uniform rotation of the liquid bridge as a solid body consists of a shifting of the bifurcation diagram toward the zone of greater nondimensional volumes [see Fig. $5(\mathrm{~d})]$. In this case. the diagram symmetry is not broken and the bifurcation is still of pitchfork type.

\section{B. Bifurcation in the range $\Lambda<\Lambda_{A}$}

Here, the bifurcation points to be analyzed are given by expression (19) with $0 \leqslant a_{1} \leqslant a \leqslant a_{2}\left[a_{1}\right.$ given by (20) and $\left.a_{2}=1.616196 \ldots\right]$. Moreover, the null space of the Frechet derivative operator is generated by the vector $\left(f_{2}, q_{2}\right)$ given by expressions (15), (21), and (22). Therefore, in the asymptotic expansions used in this section. the following values are taken:

$$
f=\frac{f_{2}}{q_{2}}=\widetilde{f}_{2}, \quad q=\mathbf{1}
$$

By solving the different order problems, the functions and coefficients taking part in the first approximation of the asymptotic expansions of $u(z), r$, and $\Psi$ are easily obtained and summarized in Appendix 2. The corresponding bifurcation equation in the neighborhood of the marginally stable equilibrium solutions is

$$
\begin{aligned}
& \frac{1}{2 \Lambda\left\|\left(\widetilde{f}_{2}, 1\right)\right\|^{2}}\left[V \overline{g_{2}}+W \overline{g_{5}}+\epsilon^{2} \overline{g_{11}}+H^{2} \overline{g_{33}}+2 H B \overline{g_{34}}\right. \\
& \left.\quad+B^{2} \bar{g}_{44}+\cdots\right]=0 .
\end{aligned}
$$

which is valid for the range $\Lambda_{C} \leqslant \Lambda \leqslant \Lambda_{A}$. The coefficients $\overline{g_{2}}, \overline{g_{5}}, \overline{g_{11}}, \overline{g_{33}}, \overline{g_{34}}$, and $\overline{g_{44}}$ are deduced from expressions (A12), (A20) and (A18), respectively.

In Fig. 6 the variation of these coefficients with the parameter $\Lambda$ is shown. Integrals appearing in $\mathrm{Eq}$. (A18) have been calculated numerically. In the neighborhood of the 

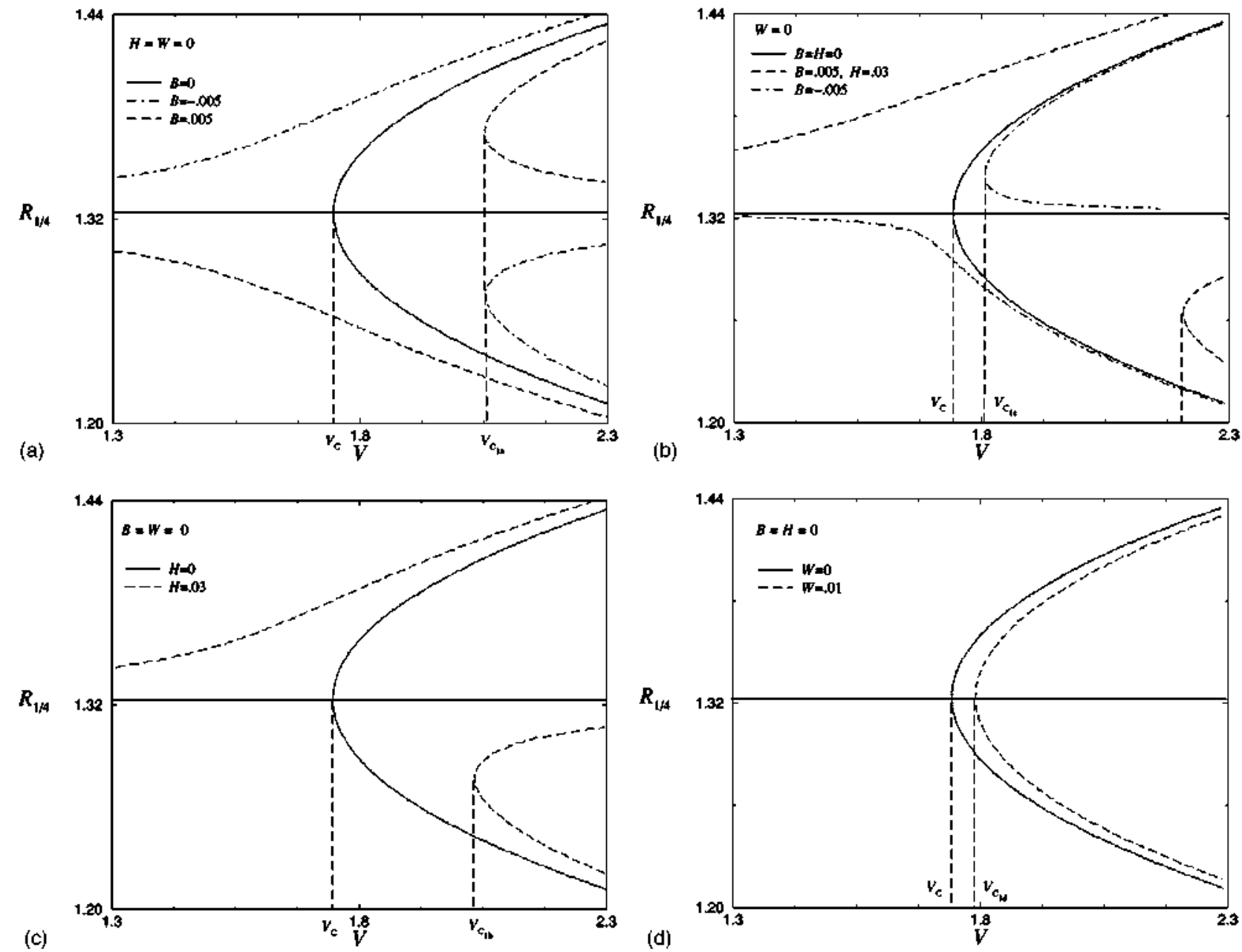

FIG. 5. Bifurcation map of a long liquid bridge $(\Lambda=4)$. The map corresponding to zero external forces is compared to those obtained for the following imperfections: small axial acceleration (a), small radii difference (b), small axial gravity and small radii difference (c), small bridge isorotation around its axis (d).

"double point" $\left(\Lambda=\Lambda_{d}\right)$, the dominant terms of the bifurcation equation are due to the small imperfections in $B$ and $H\left(g_{33}, g_{34}\right.$, and $\left.g_{44}\right)$. These coefficients have unbounded values at this point, whereas $g_{2}, g_{5}$, and $g_{11}$ remain finite.

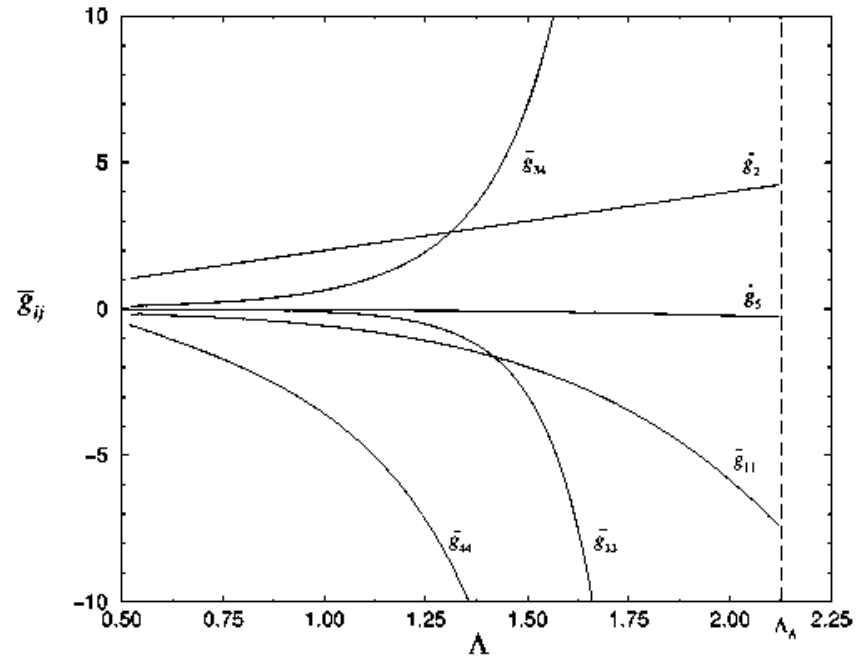

FIG. 6. Bifurcation equation coefficients as a function of slenderness, $A$ $<\Lambda_{A}$.
Therefore, near the double point $A$, higher order terms must be considered in the preceding bifurcation equation to know the effect of any of these imperfections on the minimum stable volume. By recalling the problem symmetries, the terms to be considered in this special case are those in $\epsilon V^{\prime}$. $\epsilon^{3} . \epsilon W, \epsilon H^{2}, \epsilon H B$, and $\epsilon B^{2}$.

From the bifurcation equation, it is also deduced that. when small values of $B$ and $H$ are considered. the stability limit is shifted in an amount proportional to $\left(H^{2} g_{33}\right.$ $+2 \mathrm{HBg}_{34}+\mathrm{B}^{2} g_{44}$ ). The effect of the isorotation is nearly negligible due to the small values of the ratio $g_{5} / g_{2}$.

Moreover. for a liquid bridge between two equals disks subjected to no external forces. bifurcation at the minimum volume stability limit in the range $\Lambda_{C} \leqslant \Lambda \leqslant \Lambda_{A}$ arises as a "turning point" in which the stability of the equilibrium solution changes. This behavior is shown along the curves corresponding to $H=B=W=0$ of Fig. 7, where the bifurcation diagrams in the $\left(R_{1: 4}, v\right)$ plane are plotted for $\Lambda=1.6$. Here, the shape radius at $z=-\Lambda / 2$ is approached by

$$
\begin{aligned}
R_{1 / 4}= & \bar{F}(-\Lambda / 2)+\frac{\epsilon}{q_{2}} f_{2}(-\Lambda / 2)+H u_{3}(-\Lambda / 2) \\
& +B u_{4}(-\Lambda / 2),
\end{aligned}
$$



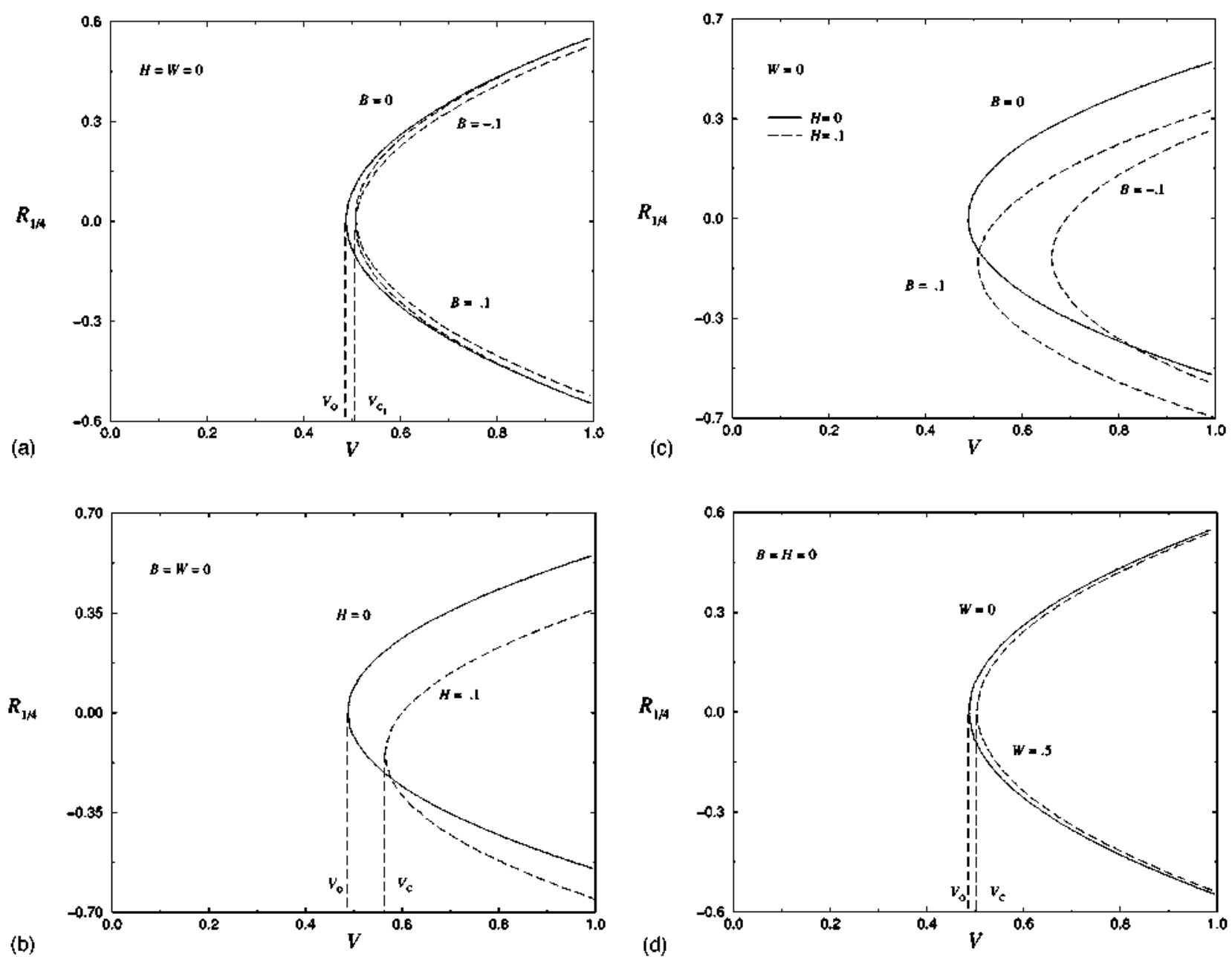

FIG. 7. Bifureation map of a short liguid bridge $(\Lambda=1.6)$. The map corresponding to zero external forces is compared to those obtained for the following imperfections: small axial acceleration (a), small radii difference (b), small axial gravity and small radii difference (c), small bridge isorotation around its axis (d).

where the functions $f_{2}(-\Lambda / 2), u_{3}(-\Lambda / 2)$, and $u_{4}(-\Lambda / 2)$ have been obtained from expressions (15), (A13), and (A15), respectively.

The presence of an imperfection, such as axial gravity or disks diameter inequality, leads to a shifting of the bifurcation diagram toward larger volumes without breaking of diagram symmetry, as shown in Fig. 7. Because of the presence of functions which are antisymmetric with respect to $z=0$ $\left(u_{3}(z)\right.$ and $\left.u_{4}(z)\right)$ in the expression of $R_{1 / 4}$. a diagram shifting along the $R_{1 / 4}$ axis also arises. In Fig. 7(c), it is easily seen that a small value of $H$ partially cancels the effect of a positive value of $B$. A nonzero value of $H$ moves the turning point toward values of the limit volume smaller than those corresponding to the same value of $B$ and $H=0\left[y_{c 1}\right.$, see Fig. 7(a)]. Both of these effects add up in the present configuration, when negative values of the Bond number are considered.

Figure 7(d) also shows that the effect of the liquid bridge isorotation is a very small shifting toward the range of greater limit volumes without any symmetry change.

\section{CONCLUSIONS}

The asymptotic behavior near the stability limits of axisymmetric liquid bridges is considered for small imperfec- tions $(H, B$, and $W)$. Actually, the effect of these small imperfections has been analyzed on the whole of the minimum volume stability limit which is critical to axisymmetric perturbations. In this way. the bifurcation to new equilibrium solutions has been analytically described in the neighborhood of those critical points using the Lyapunov-Schmidt method. The obtained results are in agreement with the numerical ones previously published.

As a preparation of the bifurcation analysis. the stability limits of axisymmetric liquid bridges held between two parallel coaxial solid equal disks in the absence of external forces have been described in detail. In this study, it has been assumed that the liquid bridge is subjected to arbitrary small perturbations, not only axisymmetric ones (the only ones considered in the most of the previous works) but also nonaxisymmetric ones. An analytical formulation in terms of elliptic variables has been obtained in order to describe the maximum and minimum volume stability limit corresponding to arbitrary liquid columns, both of them being critical to nonaxisymmetric perturbations. Besides, an analytical study of the minimum volume stability limit which is critical to axisymmetric perturbations has been performed.

When the liquid bridge becomes unstable, the breaking 
process has a very different evolution depending on the bridge slenderness. Thus, in the absence of external forces and for liquid bridge slendemess gneater than that of the "double point," $\Lambda_{s}$, the first found critical point corresponds to a subcritical "pitchfork" type bifurcation and the associated unstable mode is an odd function of $z$. However, for bridge slenderness smaller than $\Lambda_{A}$, the first critical point is a turning point and the associated function is symmetric with respect to the plane $z=0$. This fact leads one to think that, in practical cases. unstable liquid bridges break into two unequal drops in the range $\Lambda>\Lambda_{A}$ and into two equal drops for $\Lambda<\Lambda_{A}$. This behavior have been confirmed by experiments.

If axisymmetric imperfections are considered. such as small axial gravity or small inequality of the disks diameter. two unequal drops are expected as the result of the breaking. In the neighborhood of solutions with slendemesses larger than $\Lambda_{s}$, these effects shift, proportionally to $B^{2 / 3}$ and $H^{2 / 3}$, the minimum volume stability limit toward values larger than those corresponding to $H=B=0$. The bifurcation diagram symmetry is broken, and two isolated branches of solutions appear. The critical point of pitchfork type is transformed into a turning point. The larger the liquid bridge slenderness the more destabilizing the effects of nonzero $B$ and $H$. However, these destabilizing effects can cancel each other for some particular $B / H$ ratio. which is a function of the bridge slenderness. For these particular combinations of $B$ and $H$, some higher order terms should be considered in the bifurcation equation to know the imperfections effect on the minimum volume stability limit. Thus, the critical behavior arising near the stability curves of the $(\Lambda . v)$ plane has been analytically described. reproducing previous numerical results (Perales. Meseguer. and Martínez ${ }^{30}$ ).

The bifurcation equation in the neigliborhood of the marginally stable equilibrium shapes with $\Lambda<\Lambda_{d}$ shows the expected shifting of the minimum volume stability limit curve by amounts proportional to $B^{2}$ and $H^{2}$. The critical point remains as a limit point, but now the perturbed shapes are nonsymmetric with respect to the plane $z=0$.

The effect of an isorotation of the liquid bridge around its axis is a shifting proportional to $W$ of the minimum volume stability limit curve. However, the bifurcation character remains unchanged, being of "pitchfork" type for slendernesses greater than $\Lambda_{A}$ and turning points for $\Lambda<\Lambda_{A}$

\section{ACKNOWLEDGMENT}

This work has been supported by the Spanish Ministry for Science and Teclnology (MCYT) under Grant No. PNE002/2001-C.

\section{APPENDIX: EXPRESSIONS FOR THE COEFFICIENTS OF THE BIFURCATION EQUATION}

\section{Case $\Lambda>\Lambda_{A}$ (subcritical pitchfork bifurcation)}

By applying the resolubility condition (36) to the problems of order $\epsilon_{1}^{2}\left(\epsilon^{2}\right), \epsilon_{2}\left(V^{\prime}\right)$, and $\epsilon_{5}(W)$, as was anticipated from the symmetries analysis done in Sec. IV, the results $g_{11}=0, g_{2}=0$, and $g_{5}=0$ are obtained. Therefore, some higher order terms become significant in the bifurcation equation. To calculate them, the functions and parameters $u_{11}(z)$ and $r_{11}, u_{2}(z)$ and $r_{2}, u_{5}(z)$ and $r_{5}$ must be previously determined. The obtained results are

$$
\begin{aligned}
& u_{11}(z)=-\frac{1}{2} \frac{(1+\alpha \cos a)\left(1-F^{2}\right)}{(1-\alpha \cos a)\left(F^{2}+\alpha \cos a\right)} \\
& \times F\left[r_{11}(1+\alpha \cos a)-G_{e}^{1}\left(F^{2}\right)\right] \\
&+\frac{1}{2} F_{z} \int_{0}^{z} \frac{\left(1-F^{2}\right)(1+\alpha \cos a)}{\left(F^{2}+\alpha \cos a\right)(1-\alpha \cos a)} \\
& \times F\left[r_{11}(1+\alpha \cos a)-G_{e}^{2}\left(F^{2}\right)\right] d z, \\
& r_{11}=\frac{-\int_{0}^{\Lambda} H_{e}\left(F^{2}(z)\right) d z}{\sqrt{\alpha}(1+\alpha \cos a) \sin ^{2} \alpha(\mathrm{K}(a)-2 \alpha \mathrm{E}(a))},
\end{aligned}
$$

where

$$
\begin{aligned}
G_{e}^{1}\left(F^{2}\right)= & G_{e}\left(F^{2}\right) \\
= & \frac{\alpha \cos a}{F^{2}}-\frac{2(1+3 \alpha \cos a)}{(1+\alpha \cos a)\left(F^{2}+\alpha \cos a\right)} \\
+ & \frac{2 \alpha \cos a(5+\alpha \cos a)}{\left(F^{2}+\alpha \cos a\right)^{2}} \\
- & \frac{8 \alpha^{2} \cos ^{2} a(1+\alpha \cos a)}{\left(F^{2}+\alpha \cos a\right)^{3}}, \\
G_{e}^{2}\left(F^{2}\right)= & -\frac{\alpha \cos a}{F^{2}}+\frac{2(1+3 \alpha \cos a)}{(1+\alpha \cos a)\left(F^{2}+\alpha \cos a\right)} \\
& -\frac{2 \alpha \cos a\left(17+24 \alpha \cos a+3 \alpha^{2} \cos { }^{2} a\right)}{(1+\alpha \cos a)\left(F^{2}+\alpha \cos a\right)^{2}} \\
& +\frac{16 \alpha^{2} \cos ^{2} a(5+3 \alpha \cos a)}{\left(F^{2}+\alpha \cos a\right)^{3}} \\
& -\frac{48 \alpha^{3} \cos ^{3} a(1+\alpha \cos a)}{\left(F^{2}+\alpha \cos a\right)^{4}} \\
& \left.-\frac{48 \alpha^{3} \cos ^{3} a(1+\alpha \cos a)^{2}}{\left(F^{2}+\alpha \cos a\right)^{4}}\right] \\
& +\frac{\left(1-F^{2}\right)}{\left(F^{2}+\alpha \cos a\right)}\left[\frac{\alpha \cos a(7-\alpha \cos a)}{(1+\alpha \cos a)}\right. \\
& -\frac{\alpha \cos a}{F^{2}+\frac{2(1+9 \alpha \cos a)}{\left(F^{2}+\alpha \cos a\right)}} \\
& \\
H_{e}\left(F^{2}\right)= & \left(F^{2}+\alpha \cos a\right)^{2} \\
&
\end{aligned}
$$$$
u_{2}(z)=-\frac{r_{2}}{2} \frac{(1+\alpha \cos a)^{2}\left(1-F^{2}\right)}{(1-\alpha \cos a)\left(F^{2}+\alpha \cos a\right)} F
$$$$
+\frac{r_{2}}{2} F_{z} \int_{0}^{z} \frac{(1+\alpha \cos a)^{2}\left(1-F^{2}\right)}{(1-\alpha \cos a)\left(F^{2}+\alpha \cos a\right)} d z,
$$ 


$$
\begin{gathered}
r_{2}=\frac{2(\mathrm{~K}(a) \cos a+\mathrm{E}(a)) \alpha}{(1+\alpha \cos a)^{3}(\mathrm{~K}(a)-2 \alpha \mathrm{E}(a))}, \\
\begin{aligned}
u_{5}(z)= & -\frac{1}{2} \frac{(1+\alpha \cos a)^{2}\left(1-F^{2}\right)}{(1-\alpha \cos a)\left(F^{2}+\alpha \cos a\right)} F\left[r_{5}+\frac{1+F^{2}}{4}\right] \\
& +\frac{F_{z}}{2} \int_{0}^{z} \frac{(1+\alpha \cos a)^{2}\left(1-F^{2}\right)}{(1-\alpha \cos a)\left(F^{2}+\alpha \cos a\right)} \\
& \times\left[r_{5}+\frac{1+3 F^{2}}{4}\right] d z, \\
r_{5}= & \frac{-1}{12(\mathrm{~K}(a)-2 \alpha \mathrm{E}(a))}\left[\left(7-4 \sin ^{2} a\right)(\mathrm{K}(a)\right. \\
& -2 \alpha \mathrm{E}(a))+\alpha \mathrm{E}(a)] .
\end{aligned}
\end{gathered}
$$

The coefficients $g_{3}, g_{4}, g_{12}, g_{15}$, and $g_{111}$ are then obtained after applying the resolubility condition (36) to the corresponding problems. The results are shown in Table $\mathbf{I}$.

Limit for $a=0$ (cylindrical liquid column. $\cos a=\mathbf{1}, \Lambda$ $=\pi, v=1$ ). In the neighborhood of this point, the following asymptotic expansions hold:

$$
\begin{aligned}
& \mathrm{K}(a)=\frac{\pi}{2}\left(1+\frac{\sin ^{2} a}{4}+\frac{9}{64} \sin ^{4} a+\cdots\right) . \\
& \mathrm{E}(a)=\frac{\pi}{2}\left(1-\frac{\sin ^{2} a}{4}-\frac{3}{64} \sin ^{4} a+\cdots\right), \\
& \cos a=1-\frac{\sin ^{2} a}{2}+\cdots \\
& \mathrm{dn}^{2} u=1-\sin ^{2} a \sin ^{2} u .
\end{aligned}
$$

By introducing these expansions into the expressions of the bifurcation equation coefficients (see Table I), the following approximations result:

$$
\begin{aligned}
& g_{12} \cong \frac{1}{2}, \quad g_{111} \cong \frac{-3 \sin ^{4} a}{32}, \quad g_{3} \cong \frac{-8}{\pi \sin ^{2} a}, \\
& g_{4} \cong \frac{8}{\sin ^{2} a}, \quad g_{15} \cong \frac{-1}{2} .
\end{aligned}
$$

Therefore, the asymptotic behavior of the bifurcation equation in the neighborhood of the critical cylinder is $\frac{4}{\sin ^{2} a}\left[\left(\frac{\epsilon \sin ^{2} a}{4}\right)(V-W)-\frac{3}{2}\left(\frac{\epsilon \sin ^{2} a}{4}\right)^{3}-\frac{2}{\pi} H+2 B\right]=0$.

The different terms of this equation were already obtained by Meseguer, ${ }^{12}$ Vega and Perales, ${ }^{22}$ and Martine ${ }^{23}$ in the limit case $v=1, \Lambda=\pi$, with the eigenfunction $\sin z$. For $a \leqslant 1$,

$$
\begin{aligned}
\frac{F z}{\sin z} & =\frac{-\sin ^{2} a \operatorname{sn} u \operatorname{cn} u /\left(\operatorname{dn}^{2} u+\cos a\right)}{\sin (u \cos a+\mathrm{E}(a \cdot \operatorname{am} u))} \\
& \cong \frac{-\sin ^{2} a \operatorname{sn} u \operatorname{cn} u}{2 \sin (u+u)} \cong-\frac{\sin ^{2} a}{4}+\cdots .
\end{aligned}
$$

Thus, to reproduce the results of such references, bifurcation equation (A10) must be divided by the factor (A11) and the parameter $\epsilon$ must be multiplied by the same quantity.

\section{Case $\Lambda<\Lambda_{A}$ (turning point)}

By applying the resolubility condition (36) to the problem of order $\epsilon_{2}(\mathrm{~V})$,

$$
g_{2}=\frac{2 \Lambda}{2 \Lambda\left\|\left(\tilde{f}_{2}, 1\right)\right\|^{2}} .
$$

where

$$
\left\|\left(\widetilde{f}_{2}, 1\right)\right\|^{2}=\left\langle\left(\widetilde{f}_{2}, 1\right),\left(\widetilde{f}_{2}, 1\right)\right\rangle=\frac{1}{\Lambda} \int_{-\Lambda}^{\Lambda} F \widetilde{f}_{2}{ }^{2} d z+1 .
$$

The problems of order $\epsilon_{3}(H)$ and $\epsilon_{4}(B)$ lead to the expected results $g_{3}=0$ and $g_{4}=0$, and thus higher order terms are needed. So, $u_{3}(z)$ and $r_{3}, u_{4}(z)$ and $r_{4}$, must be calculated, the results being

$$
\begin{aligned}
& u_{3}(u)=-\frac{\operatorname{dn}^{2} u_{2}+\cos a}{\operatorname{sn} u_{2} \operatorname{cn} u_{2}} \frac{\operatorname{sn} u \operatorname{cn} u}{\operatorname{dn}^{2} u+\cos a}, \\
& r_{3}=0, \\
& u_{4}(u)=-\frac{\operatorname{sn} u \operatorname{cn} u}{\operatorname{dn}^{2} u+\cos a}\left[\bar{h}_{1}(u)-\bar{h}_{1}\left(u_{2}\right)\right], \\
& r_{4}=0,
\end{aligned}
$$

where

$$
\begin{aligned}
\bar{h}_{1}(u)= & \frac{(1+\cos a)^{3} \alpha^{3 / 2}}{6 \sin ^{4} a}\left[\frac{\left(1+6 \cos a+\cos ^{2} a\right)}{2}\left[\cos ^{2} a(u-\mathrm{K}(a))^{2}+(\mathrm{E}(a, \operatorname{am} u)-\mathrm{E}(a))^{2}-\operatorname{dn}^{2} u\right]\right. \\
& -\cos a\left(3+2 \cos a+3 \cos ^{2} a\right)(u-\mathrm{K}(a))(\mathrm{E}(a, \operatorname{am} u)-\mathrm{E}(a))-2 \sin ^{4} a \int_{K}^{u}(\mathrm{E}(a, \operatorname{am} x)-\mathrm{E}(a)) d x \\
& +\frac{\operatorname{dn} u \operatorname{sn} u}{\operatorname{cn} u}\left[\cos ^{2} a(1+3 \cos a)(u-\mathrm{K}(a))-\left(2+3 \cos a-2 \cos ^{2} a\right)(\mathrm{E}(a, \operatorname{am} u)-\mathrm{E}(a))\right] \\
& \left.-\frac{\operatorname{dn} u \operatorname{cn} u}{\operatorname{sn} u}\left[\cos a(3+\cos a)(u-\mathrm{K}(a))+\left(1-3 \cos a-2 \cos ^{2} a\right)(\mathrm{E}(a, \operatorname{am} u)-\mathrm{E}(a))\right]\right]
\end{aligned}
$$


Notice that $\overline{h_{1}}(u)$ is a symmetric function with respect to $u=\mathrm{K}(a)$.

For $i$ and $j$ taking values 1,3 , and 4, the resolubility condition (36) applied to problems (34) leads to

$$
\begin{aligned}
g_{i j}= & -\frac{1}{2 \Lambda\left\|\left(\tilde{f}_{2}, 1\right)\right\|^{2}}\left[r_{i} \int_{-\Lambda}^{\Lambda} \widetilde{f}_{2} u_{j} d z+r_{j} \int_{-\Lambda}^{\Lambda} \widetilde{f}_{2} u_{i} d z\right. \\
& +\int_{-\Lambda}^{\Lambda} u_{j} u_{j} d z-\Delta_{i, 4} \int_{-\Lambda}^{\Lambda} z \widetilde{f}_{2} u_{i} d z \\
& +\frac{1}{2} \int_{-\Lambda}^{\Lambda} \frac{1}{\left(1+F_{z}^{2}\right)^{3 / 2}}\left[u_{i z}\left(\tilde{f}_{2 z} u_{j}-\tilde{f}_{2} u_{j z}\right)\right. \\
& \left.\left.+u_{j z}\left(\tilde{f}_{2 z} u_{i}-\tilde{f}_{2} u_{i z}\right)\right] d z+3\left(J_{1}-J_{z}\right)\right] .
\end{aligned}
$$

Quantities $J_{1}$ and $J_{2}$ are

$$
J_{1}=\int_{-\Lambda}^{\Lambda} \frac{F F_{.} \widetilde{f}_{z z} u_{i z} u_{j z}}{\left(\mathbf{I}+F_{z}^{2}\right)^{5 / 2}} d z, \quad J_{2}=\int_{-\Lambda}^{\Lambda} \frac{\widetilde{f}_{2 z} u_{j} u_{i z}}{\left(\mathbf{I}+F_{z}^{2}\right)^{3 / 2}} d z
$$

except in the case $i=3, j=4$, where

$$
J_{2}=\frac{1}{2} \int_{-\Lambda}^{\Lambda} \frac{\tilde{f}_{2 z}\left(u_{3} u_{4 z}+u_{4} u_{3 z}\right)}{\left(1+F_{z}^{2}\right)^{3 / 2}} d z
$$

As the integrands in the cases $i=1$ and $j=3$ or $j=4$, are odd functions of $z$ and $r_{1}=r_{3}=r_{4}=0$. the expected results $g_{14}=0$ and $g_{13}=0$ follow.

Finally, by imposing the resolubility condition to the problem of order $\epsilon_{5}(W)$, the coefficient

$$
g_{5}=\frac{-1}{2 \Lambda\left\|\left(\tilde{f}_{2} \cdot 1\right)\right\|^{2}} \int_{-\Lambda}^{\Lambda} F^{3} \tilde{f}_{2} d z
$$

results. The above-mentioned integral can be expressed as

$$
\begin{aligned}
\int_{-\Lambda}^{\Lambda} F^{3} \widetilde{f}_{2} d z= & \frac{\alpha^{2}}{12 q_{2} \sin ^{2} a}\left\{\left[3 \cos ^{2} a\left(1+\cos ^{2} a\right) B-\cos ^{2} a\left(1-4 \cos ^{2} a\right) Q\right] \Delta u-\left[6\left(1-\cos ^{2} a+\cos ^{4} a\right) B\right.\right. \\
& \left.+\left(3-13 \sin ^{2} a+8 \sin ^{4} a\right) Q\right] \Delta E-2 \sin ^{2} a \operatorname{dn} u_{2} \operatorname{sn} u_{2} \operatorname{cn} u_{2}\left[-3\left(2 \operatorname{dn}^{2} u_{2}+\left(1+\cos ^{2} a\right)\right) B\right. \\
& \left.\left.+\left(-3 \operatorname{dn}^{2} u_{2}+1-4 \cos ^{2} a\right)\right] Q-\frac{3}{\alpha^{2}}\left[\left(\left(1+\cos ^{2} a\right) B+Q\right) \Delta u-(2 B+Q) \Delta E\right]\right\},
\end{aligned}
$$

with $q_{2}, B$, and $Q$ given by expressions (21) and (22).

'A. D. Myshkis, V. G. Babskii. N. D. Kopachevskii, L. A. Slobozahanin, and A. D. Tyupsov, Low-gravity Flwid Mechanics (Springer, Berlin, 1987).

${ }^{2}$ R. D. Gillete and R. C. Dyson, "Stability of fluid interfaces of revolution between ejual solid circular plates," Chem. Eng. J. 2, 44 (1971).

${ }^{3}$ L. A. Slobozhanin and J. M. Perales. "Stability of licuid bridges between equal disks in an axial gravity field," Phys. Fluids A 5. 1305 (1993).

${ }^{4}$ L. A. Slobozhanin and J. M. Perales, "Stability of an isorotating liquid bridge between equal disks under zero-gravity conditions." Phys. Fluids 8 , 2307 (1996).

${ }^{5}$ L. A. Slobozhanin, M. Gómez, and J. M. Perales, "Stability of liquid bridges between unequal disks under zero-gravity conditions," Microgravity Sci. Technol. 8, 23 (1995).

${ }^{6}$ I. Martinez and J. M. Perales. "Liquid bridge stability data." J. Cryst. Growth 78, 369 (1986).

${ }^{7} \mathrm{~J}$, M. Perales, A. Sanz, and D. Rivas, "Eccentric rotation of a liquid bridge," Appl. Microgravity Technol. 2. 193 (1990).

${ }^{8}$ J. Meseguer, N. A. Bezdenejuyl-h. J. M. Perales, and P. Rodriguez de Francisco, "Theoretical and experimental analysis of stability limits of non-axisynmetric liquid bridges under microgravity conditions," Microgravity Sci. Techuol. 8, 2 (1995).

${ }^{9}$ S. R. Coriell, S. C. Hardy, and M. R. Cordes. "Stability of liguid zones." J. Colloid Interface Sci. 60, 126 (1977).

${ }^{10}$ B. J. Lowry. "Modes of nonaxisymmetry in the stability of fixed contact line licuid bridges and drops," J. Colloid Interface Sci. 224. 8 (2000).

${ }^{11}$ L. A. Slobozhanin, J. I. D. Alexander, and A. H. Resuick. "Bifureation of the equilibrium states of a weightless liquid bridge," Phys. Fluids 9, 1893 (1997).

${ }^{12}$ J. Meseguer, "Stability of long liquid columus," ESA SP-222, 297 (1984).

${ }^{13}$ M. Gómez, I. E. Parra, and J. M. Perales, "On the stability limit change due to imperfections," Adv, Space Res. 16. 37 (1995).

${ }^{14}$ H. Gonzalez, F. M. J. McCluskey, A. Castellanos, and A. Barrero, "Stabilization of dielectric liquid bridges by electric fields in the absence of gravity." J. Fluid Mech. 206, 545 (1989).
${ }^{15}$ B. J. Lowry and P. H. Steen. "Stability of slender licjuid bridges subjected to axial tlows." J. Fluid Mech. 330, 189 (1997).

${ }^{16}$ L. A. Slobozhanin and J. I. D. Alexander, "Stability of an isorotating liquid bridge in an axial gravity field," Phys. Fluids 9. 1880 (1997).

${ }^{17}$ L. A. Slobozhanin and J. I. D. Alexander, "Combined effect of disk inequality and axial gravity on axisynmetric licuid bridge stability," Phys. Fluids 10, 2473 (1998).

${ }^{18} \mathrm{H}$. González and A. Castellanos, "The effect of an axial electric field on the stability of a rotating dielectric cylindrical liquid bridge," Phys. Fluids A 2. 2069 (1990).

${ }^{19}$ H. Gonzalez and A. Castellanos. "The effect of residual axial gravity on the stability of licjuid columns subjected to electric fields." J. Fluid Mech. 249, 185 (1993).

${ }^{20}$ J. M. Perales, J. Meseguer, and I. Martioez, "Mininum volume stability limits for axisymmetric licjuid bridges subject to steady axial acceleration," J. Cryst. Growth 110. 855 (1991).

21. Martinez. J. M. Perales, and M. Gónez. "Effect of axial and centrifugal forces on the stability of liquid bridges," ESA SP-333, 123 (1992).

${ }^{22}$ J. M. Vega and J. M. Perales, "Almost cylindrical isorotating liquid bridges for small Bond numbers," ESA SP-191. 247 (1983).

${ }^{23}$ I. Martinez, "Stability of axisynmetric liquid bridges." ESA SP-191, 267 (1983).

${ }^{24}$ J. F. Padday, "Profiles of axially symmetric menisci," Philos. Trans. R. Soc. London. Ser. A 269, 265 (1971).

${ }^{25}$ B. J. Lowry and P. H. Steen. "Capillary surfaces: Stability from families of equilibria with application to the licjuid bridge," Proc. R. Soc. London, Ser. A 449, 411 (1995).

${ }^{26} \mathrm{~S}$. N. Chow and J. K. Hale, Methods of Bifurcation Theory: (Springer, Berlin, 1982).

${ }^{27}$ J. M. Perales, "Non-axisymmetric effects on long liquid bridges," Acta Astronaut. 15, 561 (1986). 